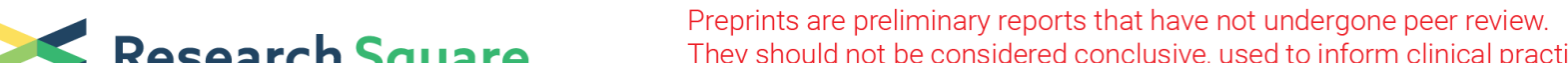 Research Square They should not be considered conclusive, used to inform clinical practice, or referenced by the media as validated information.
}

\section{Salinity-Temperature Sensor using One-Dimensional Deformed Photonic Crystal}

\section{Naim Ben Ali ( $\nabla$ naimgi2@yahoo.fr )}

University of Hail College of Engineering https://orcid.org/0000-0001-6739-679X

\section{Haitham Alsaif}

University of Hail College of Engineering

Youssef Trabelsi

King Khalid University College of Science

Muhammad Tajammal Chughtai

University of Hail College of Engineering

D. Vigneswaran

Sri Ramanathan Engineering College

Mounir Kanzari

University of Tunis El Manar: Universite de Tunis El Manar

\section{Research Article}

Keywords: Photonic crystal, Salinity Sensor, Temperature Sensor, Sensitivity, Deformation, Quality factor

Posted Date: March 11th, 2021

DOI: https://doi.org/10.21203/rs.3.rs-266806/v1

License: (c) (1) This work is licensed under a Creative Commons Attribution 4.0 International License.

Read Full License 


\title{
Salinity-Temperature Sensor using One-Dimensional Deformed Photonic Crystal
}

\author{
Naim Ben Ali ${ }^{1,2}$, Haitham Alsaif ${ }^{3}$, Youssef Trabelsi $^{4,2}$, Muhammad Tajammal Chughtai ${ }^{3}$, \\ Vigneswaran $\mathrm{D}^{5}$ and Mounir Kanzari ${ }^{2,6}$
}

\begin{abstract}
${ }^{1}$ Department of Industrial Engineering, College of Engineering, University of Ha'il, 2440, Ha'il City, Saudi Arabia

${ }^{2}$ University of Tunis El Manar, National Engineering School of Tunis, Photovoltaic and Semiconductor Materials Laboratory, 1002, Tunis, Tunisia

${ }^{3}$ Department of Electrical Engineering, College of Engineering, University of Ha'il, 2440, Ha'il City, Saudi Arabia

${ }^{4}$ College of Arts and Sciences in Muhail Asir, Physics Department, King Khalid University, Abha, Saudi Arabia.

${ }^{5}$ Department of Physics, University College of Engineering, Ramanathapuram Campus, India

${ }^{6}$ University of Tunis, Preparatory Engineering Institute of Tunis, Montfleury, 1008, Tunis
\end{abstract}

E-mail: naimgi2@yahoo.fr

\begin{abstract}
In this paper, new salinity and temperature sensor according to deformed one-dimensional photonic structure is proposed. The structure is constructed by alternating the couple of layers Air/Fused-Silica P-times. In the middle of the structure, a cavity containing the seawater is inserted to mesure its salinity and temperature. The Transfer Matrix Method (TMM) is used to simulate the wave-transmittance spectra. It is showed that, the quality factor (Q-factor) of the resonance peaks depends to the repetitive number $(P)$ of layers. After that, the thickness of the layers is deformed by changing the deformation degree $(h)$. The parameters $P$ and $h$ are optimized to get the maximal Q-factor with the minimal number of layers and structure's thickness. The best sensitivity $S_{S}$ of the proposed salinity sensor is $558.82 \mathrm{~nm} / \mathrm{RFIU}$ with a detection limit of 0.0034 RFIU. In addition, the best sensitivity $S_{T}$ of the designed temperature sensor is $600 \mathrm{~nm} / \mathrm{RFIU}$ with a detection limit of 0.0005 RFIU.
\end{abstract}

Keywords: Photonic crystal; Salinity Sensor; Temperature Sensor; Sensitivity; Deformation; Quality factor. 


\section{Introduction}

The electronic sensing devices in industrial and biological fields become a necessity nowadays. They had evolved steadily since the invention of electricity, the development of the control circuits as well the electronic chips. The health of living organisms such as human, plants and animals depends on the quality of water (like the non-existence of bacteria and the low level of mineral salts) [1]. Consequently, the necessity to develop an accurate sensing devices are requested for salinity detection [1]. Until now, these electronic devices are sensitive to the surrounding factors like electromagnetic field, heat and humidity. In addition, their accuracy is affected by the called "Joule effect" [2], which is known by the raise of the electronic devices temperature due to the flow of electrons inside them. In addition, the portable ones of these devices have a high disposable batteries consumption, which in turn is considered a source of pollution to the environment.

Photonic crystals also known photonic band gap materials are made by alternating two or more different materials. They represent the optical analogy to a crystal lattice, where atoms or molecules are periodically arranged and the periodic potential introduces gaps into the energy band structure of the crystal [3-8]. There are three different families of photonic structure, according to the direction of materials alternation, namely one-dimensional (1D), twodimensional (2D) and three-dimensional (3D) structures. In addition, the alternation of materials can be periodic or quasi-periodic (where the alternation of materials follows mathematical sequences). The emergence of these photonic structures [3-8] permits to discard several problems of the olds electronic devices such as the called Joule effect [2]. These structures represent a serious opportunity for researchers to study and improve their properties to be suitable in sensing applications. The photonic sensing devices are known by their accurate and precise response [9-11] also they have less energy consumption with rapid response because photons are faster than electrons (photons displaces with the speed $3 * 10^{8} \mathrm{~m} / \mathrm{s}$ ) [9].

Some of the previous researches were interested in studying photonic structures for sensing application. For example, the papers of D. Vigneswarana et al. [1] and Sameeha R. Qutb et al. [26] studied the photonic structures to measure salinity and temperature of water. The research of Harikesavan Thenmozhi et al. [12] presents optical glucose sensor. Furthermore, the paper of Francis Segovia-Chaves et al. [13] studied temperature and pressure sensors. Ida Pavlichenko et al. [14] proposed photonic crystal as temperature and humidity sensors. In addition, Arafa H Aly 
et al. [15] studied hemoglobin sensor and N. R. Ramanujam et al. [16] used these optical devices for early detection of several types of cancer cells.

\section{Problem formulation}

\subsection{Photonic structure Design}

To simulate the wave transmittance through a photonic structure contains a seawater layer as defect; the TMM (introduced by Yeh and Yariv [17]) is deployed. The sensitivity of the optical properties to salinity and temperature variations of seawater is studied. The studied photonic structure is constructed by alternating the Air (A) and the Fused Silica (F) as two elementary layers, and at the middle of the structure we find a cavity containing the seawater (S), which we want to measure, its salinity and temperature (see figure 1). The refractive index of Air is $n_{A}=1$ and the refractive index of the fused silica $\left(n_{F}\right)$ as function of wavelength and temperature is determined, via the Sellmier's equation $[1,22]$ :

$$
\begin{gathered}
n_{F}^{2}(\lambda, T)=\left(1.31552+6.90754 * 10^{-6} T\right)+\frac{\left(0.788404+23.5835 * 10^{-6} T\right) \lambda^{2}}{\lambda^{2}-\left(0.0110199+0.584758 * 10^{-6} T\right)} \\
+\frac{\left(0.91316+0.548368 * 10^{-6} T\right) \lambda^{2}}{\lambda^{2}-100}(1)
\end{gathered}
$$

Here $\lambda$ and $T$ represent the free space wavelength $(\mu m)$ and the temperature degree $\left({ }^{\circ} \mathrm{C}\right)$ respectively. The thicknesses $d_{A, F}$ of the Air and Fused silica layers, fulfill the Bragg condition $n_{A} * d_{A}=n_{F} * d_{F}=\frac{\lambda_{0}}{4}$, where $\lambda_{0}=1.573 \mu \mathrm{m}$ is the reference wavelength of the structure. The refractive index $n_{S}$ of the seawater cavity defect depends on the probing wavelength $\lambda(\mathrm{nm})$, the salinity percentage $S(\%)$ and the temperature degree $T\left({ }^{\circ} \mathrm{C}\right)[1,23$, and 24]:

$$
\begin{aligned}
n_{S}(S, T, \lambda)= & 1.3140+\left(1.779 * 10^{-4}-1.05 * 10^{-6} T+1.6 * 10^{-8} T^{2}\right) S-2.02 * 10^{-6} T^{2} \\
& +\frac{15.868+0.01155 S-0.00423 T}{\lambda}-\frac{4382}{\lambda^{2}}+\frac{1.1455 * 10^{-6}}{\lambda^{3}}(2)
\end{aligned}
$$

$n_{S}$ is represented in refractive index units (RFIU). The thicknesses $d_{S}$ of the seawater cavity obey to the condition $n_{s} * d_{s}=\lambda_{0}$. 


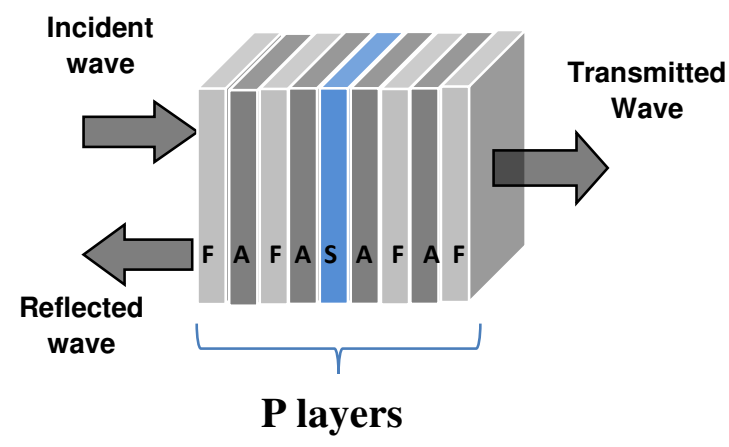

Fig. 1. Schematic representation showing periodic photonic crystal with middle cavity containing the seawater, where $\mathrm{F}$ is the fused silica layer, $\mathrm{A}$ is the air layer and $\mathrm{S}$ is the seawater layer.

\subsection{Quality Factor of resonance peak}

The quality factor (Q-factor) of a resonance peak given by a defect through a multilayer photonic structure is a measurement (without unit) that determine the central spectral position of the resonator relative to its wavelengths-bandwidth [18-21].

$$
Q=\frac{\lambda_{p i c}}{\Delta \lambda}
$$

Where $\lambda_{\text {pic }}$ is the central-wavelength of the resonance-peak and $\Delta \lambda$ represents his Full Width at Half Maximum (FWHM) [18-21].

\section{Results and discussion}

\subsection{Optimization of Q-factor and the intensity of the transmittance resonance peak}

In this part we study the effect of the structure layers number (P vary from 8 to 50) on the Qfactor then for the next parts we will keep the P-value which gives the best Q-factor. Here the salinity of the seawater is fixed at $50 \%$ and the temperature at $\mathrm{T}=25^{\circ} \mathrm{C}$ (room temperature).

From Fig. 2, we remark the presence of two transmittance peaks the first one located at $1.477 \mu \mathrm{m}$ and the second one at $1.774 \mu \mathrm{m}$. The FWHM of the first transmittance peak is $9.62 *$ $10^{-4} \mu \mathrm{m}$ and the FWHM of the second one is $6.07 * 10^{-3} \mu \mathrm{m}$. So the quality factor of the first and second peaks are $Q_{1}=1534$ and $Q_{2}=292$ respectively. Also when we change the layer number of the structure $(P)$, the first peak keep the best quality factor, therefore for the rest of the study we will concentrate on the first peak to study its sensibility to the salinity and the temperature. 
Table 1 and Fig. 3 show the variation of the structure thickness, the transmittance peak intensity and the Q-factor as function of the layer number $P$. Here it is clear that the peak intensity still upper 0.8 for $P$ varying from 8 to 56 layers, but from 62 layers, the transmittance peak disappear. In addition, the quality factor $Q$ has become more important from $P=50$ layers and the best $\mathrm{Q}$ value is obtained with this number $(P=50)$. Therefore, for the rest of the study we will keep the structure layers number $(P)$ fixed at 50 layers.

From the Table 1, it is clear that the transmittance peak intensity takes the value 0.8111 for $P=50$ layers, so in the next step we will try to improve the maximum value of the transmittance peak intensity, by applying a deformation in the structure layers thickness. The initial optical thickness of fused silica (F) and air (A) layers is $X_{0 j}=n_{A} * d_{A}=n_{F} * d_{F}=\frac{\lambda_{0}}{4}$ and the initial optical thickness of seawater $(\mathrm{S})$ cavity is $Y_{0 j}=n_{s} * d_{s}=\lambda_{0}$. Where $j$ defines the $j^{\text {th }}$ layer-position in the photonic structure. By applying the deformation law, the optical thickness of layers varies according to the deformation degree $(h)$ and the layer position $\left(j^{t h}\right)$. Therefore for $j \geq 1$, the new optical thickness of fused silica (F), air (A) and seawater (S) layers after deformation takes the forms $X_{0 j}^{\prime}=X_{0 j} *\left[j^{h+1}-(j-1)^{h+1}\right]$ and $Y_{0 j}^{\prime}=Y_{0 j} *\left[j^{h+1}-(j-1)^{h+1}\right]$ respectively [5, 25]. Fig.4 and Fig.5 illustrate the variation of the transmittance peak intensity and the Q-factor as function of the deformation degree $(h)$. It is clear that by increasing $h$ from 0 to 0.03 , the best intensity and Q-factor are found for $h=0.01$ (The values of the transmittance peak intensity and the Q-factor are 0.976 and 15060 respectively). Also with the rising of the deformation degree (h), we can remark the shift of the photonic band gap (PBG), and transmittance peak toward the high wavelengths. This physical phenomenon is due to the increase of the structure's geometric thickness $(d)$ from $17.846 \mu \mathrm{m}$ to $20.13 \mu \mathrm{m}$ when $h$ varying from 0 to 0.003 . Therefore, for the rest of this study we will keep a deformed structure with $h=0.01$. 


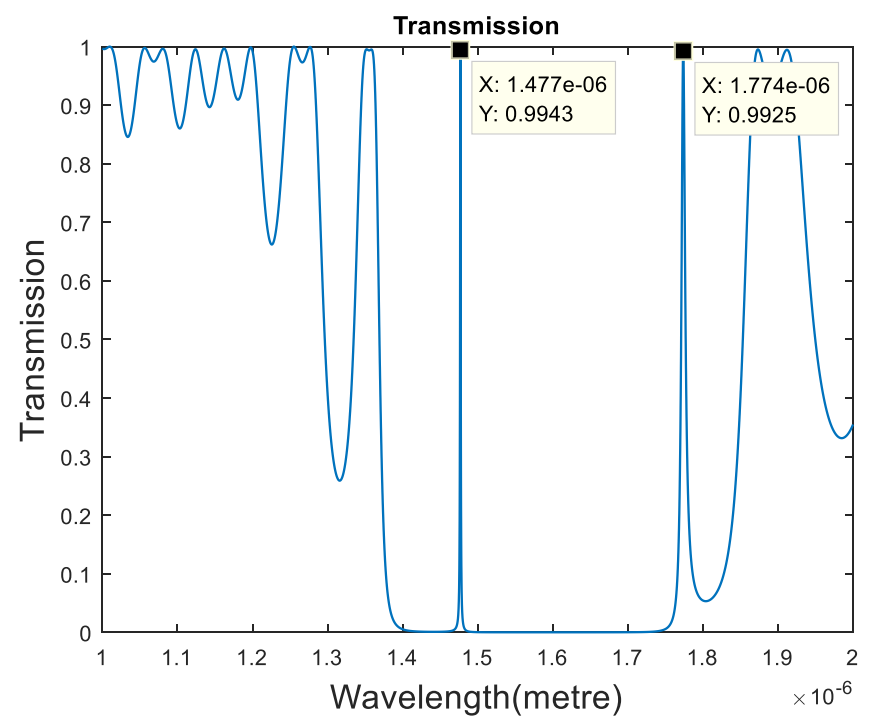

Fig. 2. Transmittance spectrum for periodic photonic structure with 36 alternated layers of fused silica and air and with middle cavity filled by seawater have $50 \%$ salinity and at room temperature.

Table 1: Variation of the peak intensity, peak wavelength, peak FWHM and the Q- factor for different values of $\mathrm{P}$.

\begin{tabular}{|c|c|c|c|c|c|}
\hline $\begin{array}{c}\text { Number } \\
\text { of } \\
\text { Layers } \\
(\mathbf{P})\end{array}$ & $\begin{array}{c}\text { Structure } \\
\text { thickness } \\
(\mu \mathrm{m})\end{array}$ & $\begin{array}{c}\text { Transmittance } \\
\text { Peak } \\
\text { Intensity }\end{array}$ & $\begin{array}{c}\text { Peak } \\
\text { wavelength } \\
(\mu \mathrm{m})\end{array}$ & $\begin{array}{c}\text { FWHM } \\
(\mu \mathrm{m})\end{array}$ & $\begin{array}{c}\text { Quality } \\
\text { Factor } \\
(\mathbf{Q})\end{array}$ \\
\hline 8 & 3.966 & 0.9935 & 1.4492 & 0.1298 & 11.164 \\
\hline 14 & 5.8463 & 0.9350 & 1.4752 & 0.0379 & 38.923 \\
\hline 20 & 7.9662 & 0.9941 & 1.4733 & 0.0123 & 119.780 \\
\hline 26 & 9.8463 & 0.9381 & 1.4838 & 0.0045 & 329.733 \\
\hline 32 & 11.966 & 0.9933 & 1.47766 & 0.0018 & 820.922 \\
\hline 38 & 13.846 & 0.9239 & 1.4850 & 0.0007 & 2121.429 \\
\hline 44 & 15.966 & 0.9547 & 1.4771 & 0.0003 & 4923.667 \\
\hline 50 & 17.846 & 0.8111 & 1.4852 & 0.0001 & 14852 \\
\hline 56 & 19.966 & 0.9000 & 1.4772 & 0.0001 & 14772 \\
\hline 62 & 21.846 & 0.0250 & 1.4852 & - & - \\
\hline 68 & 23.966 & 0.0310 & 1.4772 & - & - \\
\hline
\end{tabular}



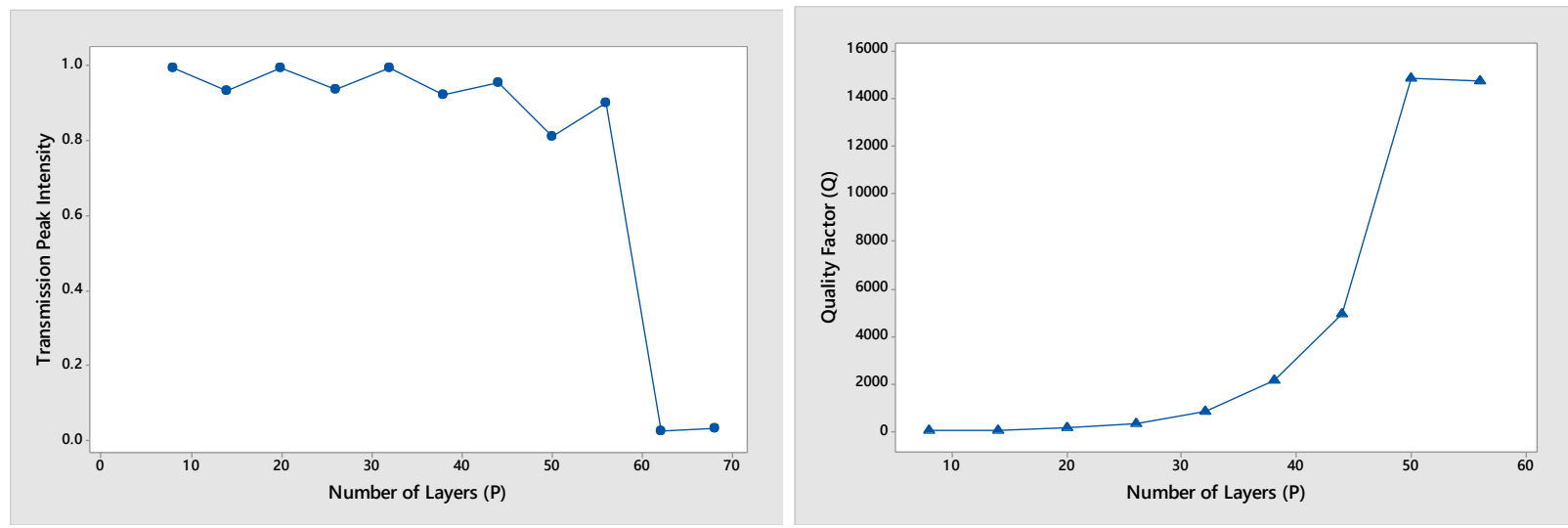

Fig. 3. Variation of the transmittance peak intensity and the quality factor as function of the layer number $(\mathrm{P})$ of photonic structure with $\mathrm{P}$-alternated layers of fused silica and air and with middle cavity filled by seawater of $50 \%$ salinity and at room temperature.
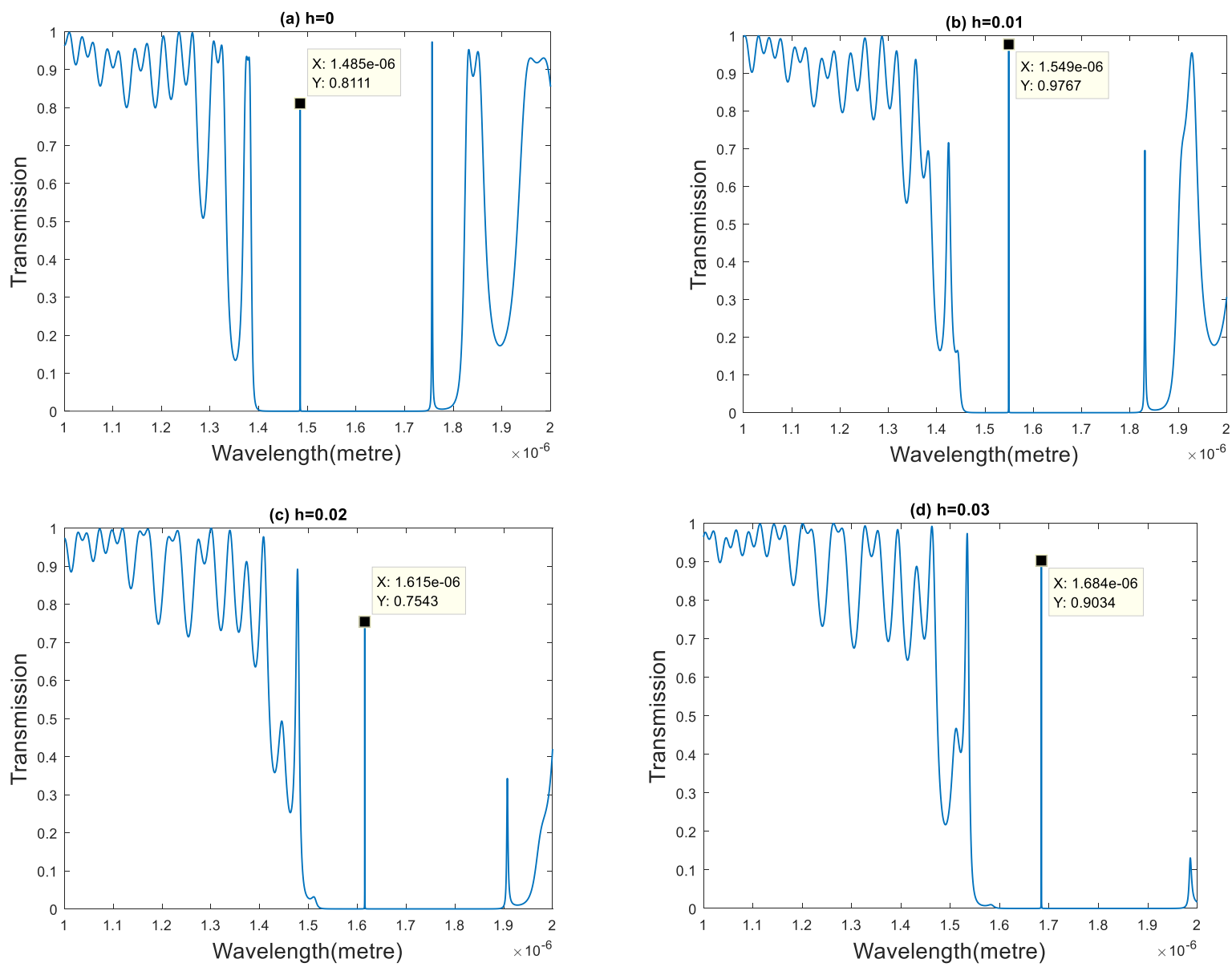

Fig. 4. Variation of the transmittance peak intensity as function of the deformation degree $(h)$ for photonic structure with 50-alternated layers of fused silica and air and with middle cavity filled by seawater of $50 \%$ salinity and at room temperature. 


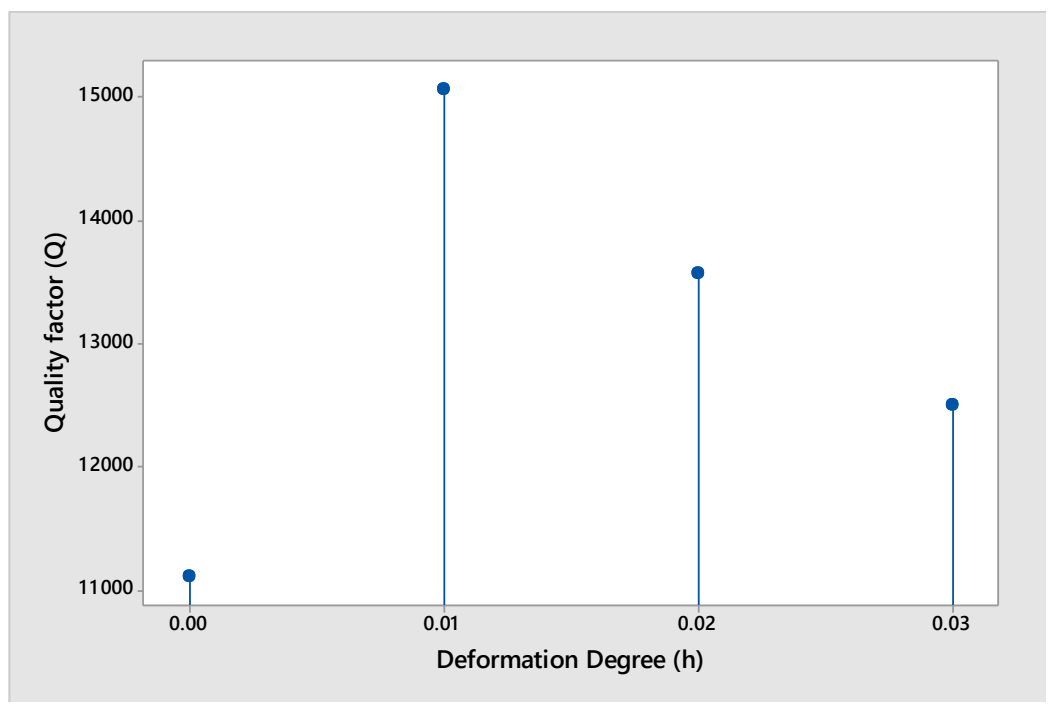

Fig. 5. Variation of Q-factor as function of the deformation degree $(h)$ for photonic structure with 50-alternated layers of fused silica and air and with middle cavity filled by seawater of $50 \%$ salinity and at room temperature.

\subsection{Salinity Sensing}

In this part we study of the variation of seawater salinity at room temperature $\left(\mathrm{T}=25^{\circ} \mathrm{C}\right)$ and when the number of layer $P$ and the deformation degree $h$ are fixed at 50 and 0.01 respectively. The sensitivity to the salinity of seawater can be defined as $S_{s}=\frac{\Delta \lambda_{\text {peak }}}{\Delta n}$, where $\Delta \lambda_{\text {peak }}$ is the wavelength shift of the resonance and $\Delta n$ is the refractive index variation [1].

Table 2 reveals the seawater salt level, its refractive index units (RFIU), the resonance peak wavelength $\left(\lambda_{\text {peak }}\right)$, the sensitivity to the salinity $\left(S_{S}\right)$ and the refractive index difference $\Delta n$. In addition, Fig.6 and Fig.7 (a) show that the resonance peak position shift toward the right wavelengths when the salt level increase with an equidistance between all peaks. It is found that the wavelength shift is equal to $9.2 \mathrm{~nm}$ when the salt levels changes from 0 to $100 \%$ (see Fig.7 (b)). Furthermore, from Table 2, it is found that the best sensitivity $S_{S}$ of the proposed Salinity sensor is $558.82 \mathrm{~nm} / \mathrm{RFIU}$ at $20 \%$ salinity with a detection limit (DL) of 0.0034 RFIU.

Table 2: Variation of the RFIU, $\lambda_{\text {peak }}$ and $S_{S}$ as function of salt level.

\begin{tabular}{|c|c|c|c|c|}
\hline Salt level $(\%)$ & $(\mathrm{RFIU})$ & $\lambda_{\text {Peak }}(\mathrm{nm})$ & $S_{S}(\mathrm{~nm} / \mathrm{RIU})$ & $\Delta n(R I U) * 10^{-2}$ \\
\hline 0 & 1.3211 & 1544.3 & - & 0 \\
\hline 10 & 1.3228 & 1545.2 & 529.41 & 0.17 \\
\hline
\end{tabular}




\begin{tabular}{|l|c|c|c|c|}
\hline 20 & 1.3245 & 1546.2 & 558.82 & 0.34 \\
\hline 30 & 1.3262 & 1547.1 & 549.02 & 0.51 \\
\hline 40 & 1.3279 & 1548 & 544.12 & 0.68 \\
\hline 50 & 1.3295 & 1548.9 & 547.62 & 0.84 \\
\hline 60 & 1.3312 & 1549.8 & 544.55 & 1.01 \\
\hline 70 & 1.3329 & 1550.7 & 542.37 & 1.18 \\
\hline 80 & 1.3346 & 1551.7 & 548.15 & 1.35 \\
\hline 90 & 1.3363 & 1552.6 & 546.05 & 1.52 \\
\hline 100 & 1.3380 & 1553.5 & 544.38 & 1.69 \\
\hline
\end{tabular}

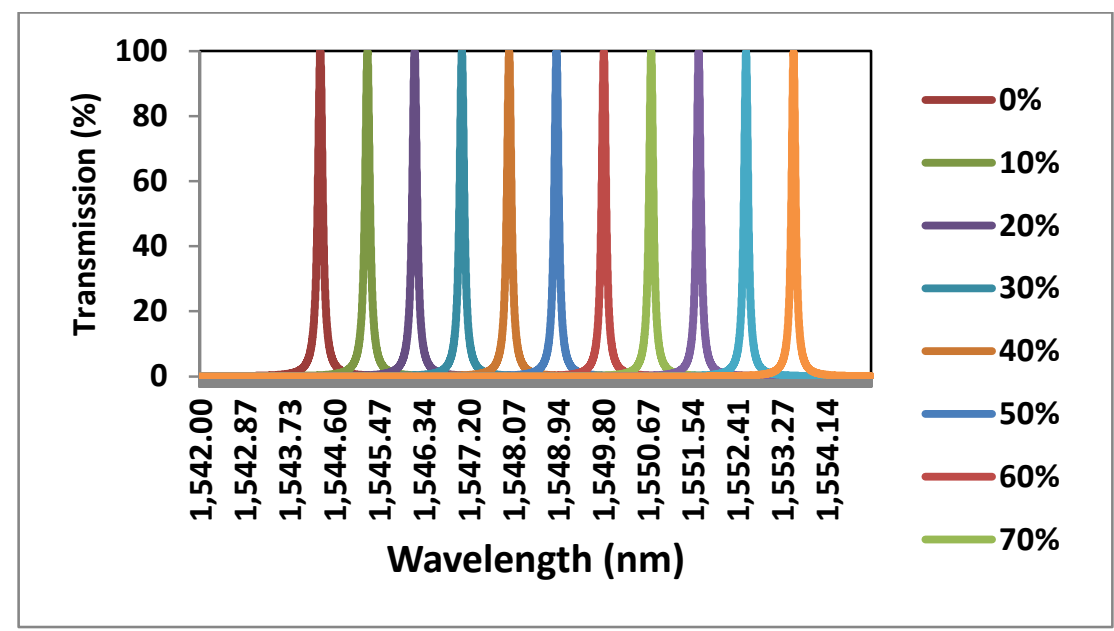

Fig. 6. Optical transmittances as function of wavelength and salt level for deformed photonic structure with 50-alternated layers of fused silica and air and with middle cavity filled by seawater at room temperature.
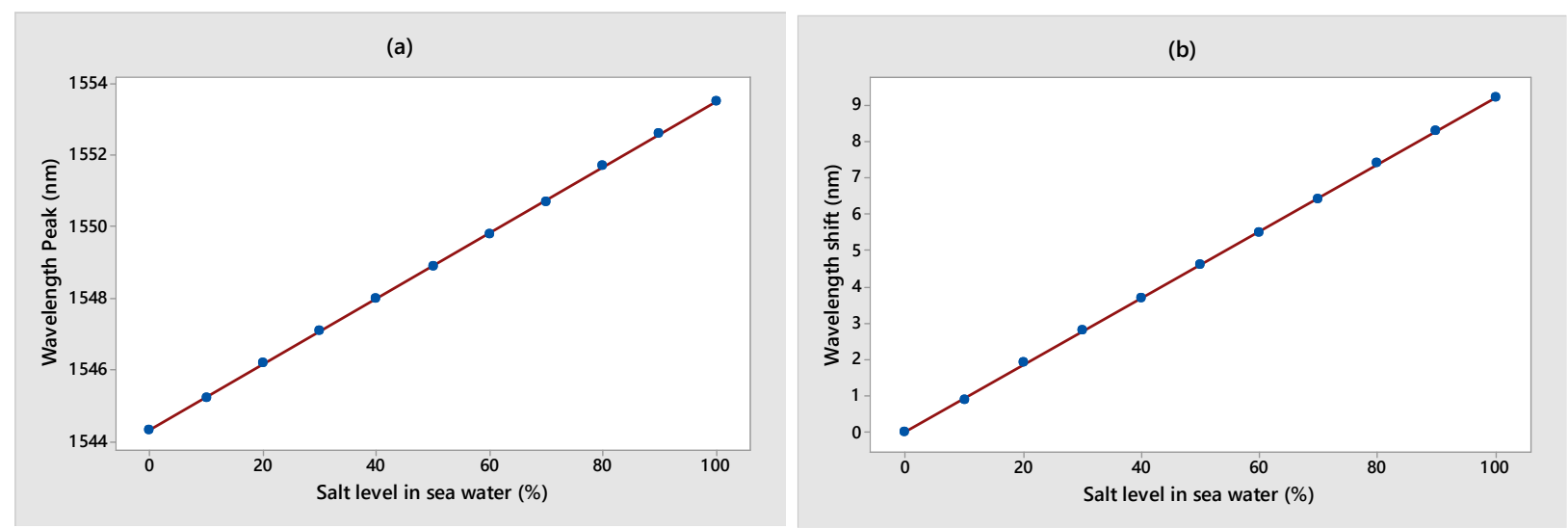

Fig. 7. (a) Wavelength of Transmittance peak and (b) Peak wavelength shift as function of salt level for deformed photonic structure with 50-alternated layers of fused silica and air and with middle cavity filled by seawater at room temperature. 


\subsection{Temperature Sensing}

In this part, we study of the variation of the seawater temperature when the number of layer $P$, the deformation degree $h$ and the standard Salinity water are fixed at 50, 0.01 and 35\% respectively. The sensitivity to the temperature of seawater is determined by the formula $S_{T}=$ $\frac{\Delta \lambda_{\text {peak }}}{\Delta n}$, where $\Delta \lambda_{\text {peak }}$ is the wavelength shift of the resonance and $\Delta n$ is the refractive index variation [1].

Table 3 shows the seawater temperature, its refractive index units (RFIU), the resonance peak wavelength $\left(\lambda_{\text {peak }}\right)$, the sensitivity to the temperature $\left(S_{T}\right)$ and the refractive index variation $\Delta n$. In addition, Fig.8 and Fig.9 (a) show that the resonance peak position shift toward the lowest wavelengths when the seawater temperature rise. In contrast to the changes in the salinity degree, the changes in seawater temperature led to changes in peak resonance locations and the distance between them. We noticed an enlargement between peaks positions when increasing the temperature and it is physically explained by the variation of the refraction index of seawater, as function of temperature. This variation is parabolic on the other hand the variation of this index as a function of salinity degree is linear. From Fig.9 (b), it is clear that the wavelength shift is equal to $10.1 \mathrm{~nm}$ when the temperature changes from 0 to $100^{\circ} \mathrm{C}$. In addition, from Table 3 , we noticed that the best sensitivity $S_{T}$ of the proposed temperature sensor is $600 \mathrm{~nm} / \mathrm{RFIU}$ when the temperature degree of the seawater is $10^{\circ} \mathrm{C}$ with a DL of $0.0005 \mathrm{RFIU}$.

Table 3: Variation of the RFIU, $\lambda_{\text {peak }}$ and $S_{T}$ as function of temperature.

\begin{tabular}{|c|c|c|c|c|}
\hline $\begin{array}{c}\text { Temperature } \\
\text { Degree }\left({ }^{\circ} \mathrm{C}\right)\end{array}$ & $(\mathrm{RFIU})$ & $\lambda_{\text {Peak }}(\mathrm{nm})$ & $S_{T}(\mathrm{~nm} / \mathrm{RIU})$ & $\begin{array}{c}\Delta n(R I U) \\
* 10^{-2}\end{array}$ \\
\hline 0 & 1.3289 & 1548.6 & - & 0 \\
\hline 10 & 1.3284 & 1548.3 & 600 & 0.05 \\
\hline 20 & 1.3275 & 1547.8 & 571.43 & 0.14 \\
\hline 30 & 1.3264 & 1547.2 & 560 & 0.25 \\
\hline 40 & 1.3250 & 1546.4 & 564.1 & 0.39 \\
\hline 50 & 1.3233 & 1545.5 & 553.57 & 0.56 \\
\hline 60 & 1.3213 & 1544.4 & 552.63 & 0.76 \\
\hline 70 & 1.3190 & 1543.1 & 555.56 & 0.99 \\
\hline 80 & 1.3164 & 1541.7 & 552 & 1.25 \\
\hline 90 & 1.3136 & 1540.2 & 549 & 1.53 \\
\hline 100 & 1.3104 & 1538.5 & 545.95 & 1.85 \\
\hline
\end{tabular}




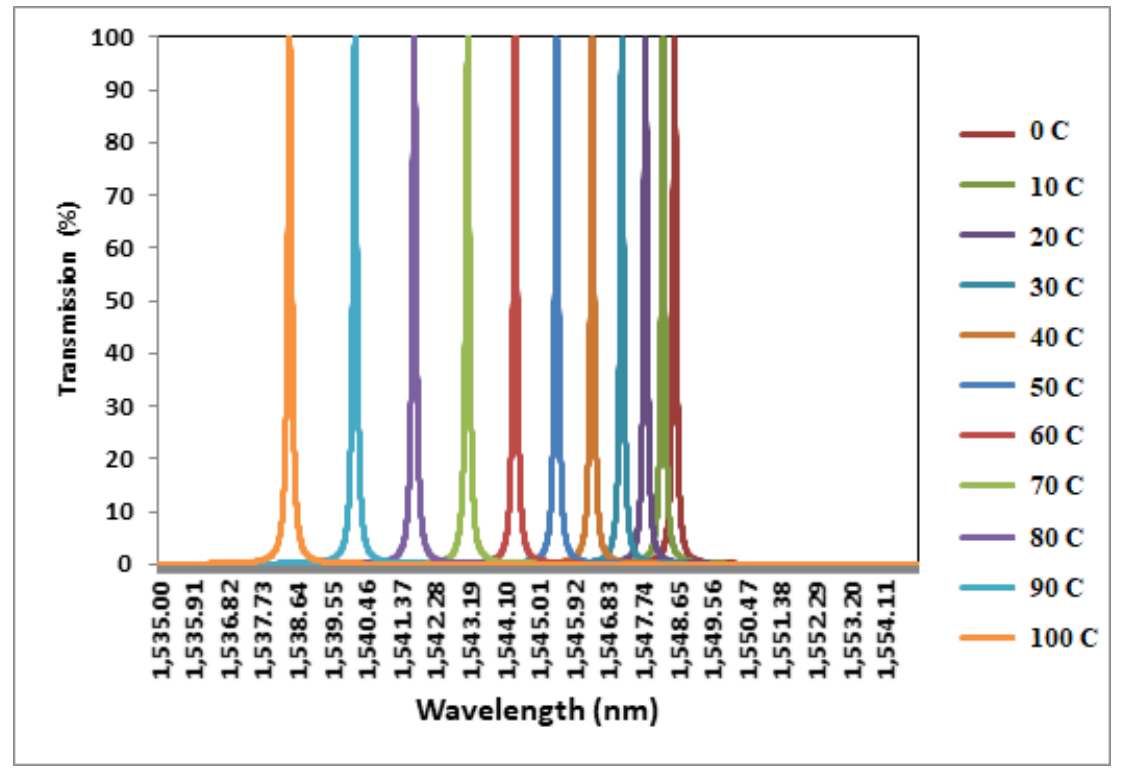

Fig. 8. Optical transmittances as function of wavelength and water temperature degree $\left({ }^{\circ} \mathrm{C}\right)$ for deformed photonic structure with 50-alternated layers of fused silica and air and with middle cavity filled by seawater at $35 \%$ salt level.
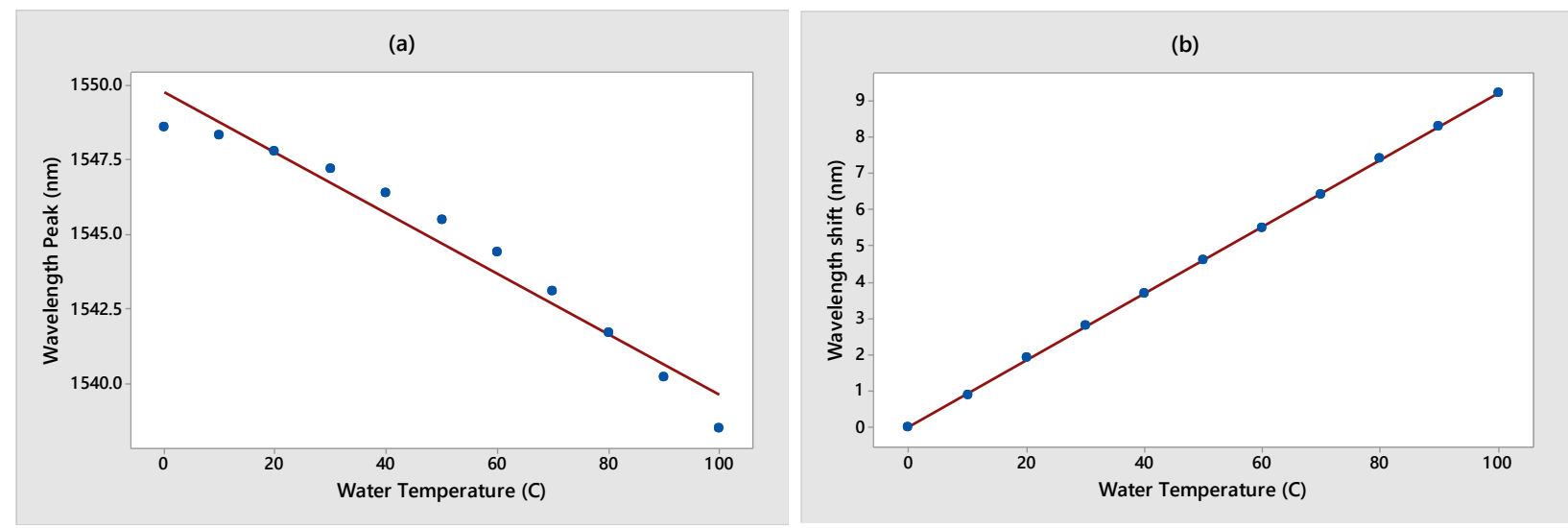

Fig. 9. (a) Wavelength of Transmittance peak and (b) Peak wavelength shift as function of water temperature degree $\left({ }^{\circ} \mathrm{C}\right)$ for deformed photonic structure with 50 -alternated layers of fused silica and air and with middle cavity filled by seawater at $35 \%$ salt level.

\section{Conclusion}

The studied deformed photonic structure represented a real opportunity to use optical circuits as sensors for the salinity and temperature of the water. As the first step, the number of layers is optimized to achieve the best quality factor $(\mathrm{Q}$-factor=14852) and after that we tried to deform the layers thickness by applying a mathematic formula. After that, the deformation degree is 
optimized to be equal to $h=0.01$ and the best transmittance peak intensity and Q-factor found are 0.976 and 15060 respectively. By changing the water salinity level from 10 to $100 \%$, we studied the sensitivity of the photonic structure. The resonance-peak-position shift toward the highest wavelengths when the salt level increase with an equidistance between all peaks. It is found that the wavelength shift is equal to $9.2 \mathrm{~nm}$ when the salt level changes from 0 to $100 \%$. The best sensitivity of the proposed salinity sensor is $S_{S}=558.82$ at $20 \%$ salinity of seawater with a DL of 0.0034 RFIU. Finally, the temperature sensitivity is studied. The resonance-peakposition shift toward the lowest wavelengths when the seawater temperature rise and an enlargement between peaks positions is remarked. The wavelength shift is equal to $10.1 \mathrm{~nm}$ when the temperature degree changes from 0 to $100^{\circ} \mathrm{C}$. The best sensitivity of the proposed temperature sensor is $S_{T}=600$ when the temperature degree of the seawater is $10^{\circ} \mathrm{C}$ with a DL of 0.0005 RFIU.

\section{Declaration}

The authors declare that they have no known competing financial interests or personal relationships that could have appeared to influence the work reported in this paper.

\section{Acknowledgment}

"This research has been funded by Scientific Research Deanship at University of Ha'il - Saudi Arabia through project number RG-20 013".

\section{References}

[1] D. Vigneswarana, N. Ayyanarb, Mohit Sharmac, M. Sumathib, M.S. Mani Rajand, K. Porsezian, "Salinity sensor using photonic crystal fiber" Sensors and Actuators A 269 (2018) 2228

[2] Bongjin Simon Mun et al., "Role of joule heating effect and bulk-surface phases in voltagedriven metal-insulator transition in VO2 crystal", Appl. Phys. Lett. 103, 061902 (2013); https://doi.org/10.1063/1.4817727

[3] V.Schmidt, Laser Growth and Processing of Photonic Devices, Woodhead Publishing Series in Electronic and Optical Materials, 2012, Pages 162-237. https://doi.org/10.1533/9780857096227.2.162

[4] R Asmi, NB Ali, M Kanzari, "Numerical investigation of light localization in generalized Thue-Morse one-dimensional photonic crystal" 
Journal of Photonics for Energy 6 (3), 034501(2016).

[5] NB Ali, M Kanzari, "omni-directional high reflectors using one-dimensional deformed quasiperiodic Cantor band gap structure at optical telecommunication wavelength band", The Mediterranean Journal of Electronics and Communications 6 (2), 1-6 (2010).

[6] Y Trabelsi, NB Ali, M Kanzari, "Tunable narrowband optical filters using superconductor/dielectric generalized Thue-Morse photonic crystals", Microelectronic Engineering 213, 41-46 (2019).

[7] K Srinivasan, NB Ali, Y Trabelsi, MSM Rajan, M Kanzari, "Design of a modified singlenegative metamaterial structure for sensing application", Optik 180 (2019) 924-931.

[8] Serpil Savci, "Investigation of Effect of Chemical Fertilizers on Environment", APCBEE Procedia 1(2012) $287-292$.

[9] NB Ali, J Zaghdoudi, M Kanzari, R Kuszelewicz "The slowing of light in one-dimensional hybrid periodic and non-periodic photonic crystals" Journal of Optics 12 (4), 045402 (2010).

[10] NB Ali, Y Trabelsi, M Kanzari "Stop band filter by using hybrid quasi-periodic one dimensional photonic crystal in microwave domain" IJMOT, 4, 195-204 (2009).

[11] Y Trabelsi, NB Ali, A Elhawil, R Krishnamurthy, M Kanzari, IS Amiri "Design of structural gigahertz multichanneled filter by using generalized Fibonacci superconducting photonic quasicrystals" Results in Physics 13, 102343 (2019).

[12] Harikesavan Thenmozhia, MuruganSenthil Mani Rajana, Veluchamy Devikaa, Dhasarathan Vigneswaranb, Natesan Ayyanarc, "D-glucose sensor using photonic crystal fiber”, Optik 145 (2017) 489-494

[13] Francis Segovia-Chaves, Herbert Vinck-Posada, "Effects of temperature, pressure and thickness on a one-dimensional

Thue-Morse photonic crystal”, Optik 203 (2020) 163887

[14] Ida Pavlichenko, Armin T. Exner, Markus Guehl, Paolo Lugli, Giuseppe Scarpa and Bettina V. Lotsch, "Humidity-Enhanced Thermally Tunable TiO2/SiO2 Bragg Stacks", J. Phys. Chem. C 116 (2012) 298-305 https://doi.org/10.1021/jp208733t

[15] Arafa H Aly,Walaa M. Nouman, S.E.-S. Abd El-Ghany, Samira M.Sallam and Abdel-Fattah B. Dawood," Theoretical studies on hemoglobin periodic structure sensor", Exp. Theo. NANOTECHNOLOGY 3 (2019) 203 - 212. 
[16] N. R. Ramanujam, I. S. Amiri, Sofyan A. Taya, Saeed Olyaee, R. Udaiyakumar, A. Pasumpon Pandian, K. S. Joseph Wilson, P. Mahalakshmi and P. P. Yupapin, "Enhanced sensitivity of cancer cell using one dimensional nanocomposite material coated photonic crystal”, Microsystem Technologies 25, (2019)189-196

[17] Yeh P. and Yariv A., Optical Waves in Crystals. A Wiley-Interscience publication, New York, 1984.

[18] N. Ben Ali, Optical Fabry-Perot filters using hybrid Periodic, Fibonacci and Cantor photonic structures, Nano Communication Networks 13 (2017) 34-42

[19] J. H. Harlow, Electric power transformer engineering, CRC Press, New York, 2012.

[20] M. Tooley, Electronic circuits: fundamentals and applications, third ed., London, 2006.

[21] J. A. French, Design and Characterization of a Tunable Fabry-Perot Filter Using an Electrooptic Modulated Spacer Layer, ProQuest, Washington, 2007.

[22] Qiang Liu, Shuguang Li, Hailiang Chen, Jianshe Li, Zhenkai Fan, High-sensitivity plasmonic temperature sensor based on photonic crystal fiber coated with nanoscale gold film, IOP, science, Appl. Phys. Exp. 8 (2015) 046701.

[23] X.H. Quan, E.S. Fry, Empirical equation for the index of refraction of seawater, Appl. Opt. 34 (1995) 3477-3480.

[24] F. Adamo, F. Attivissimo, C.G.C. Carducci, A small sensor network for sea water Quality monitoring, IEEESens.J.15(5) (2015) 2514-2522.

[25] K. Ben Abdelaziz, J. Zaghdoudi, M. Kanzari, B. Rezig, "A broad omnidirectional reflection band obtained from deformed Fibonacci quasi-periodic one dimensional photonic crystals", Journal of Optics A: Pure and Applied Optics, Vol. 7, 2005, pp. 544-549.

[26] Sameeha R. Qutb, Arafa H. Aly and Walied Sabra, "Salinity and temperature detection for seawater based on a 1D-defective photonic crystal material", IJMPB 2150012 (2020) 1-13 https://doi.org/10.1142/S0217979221500120 


\section{Figures}

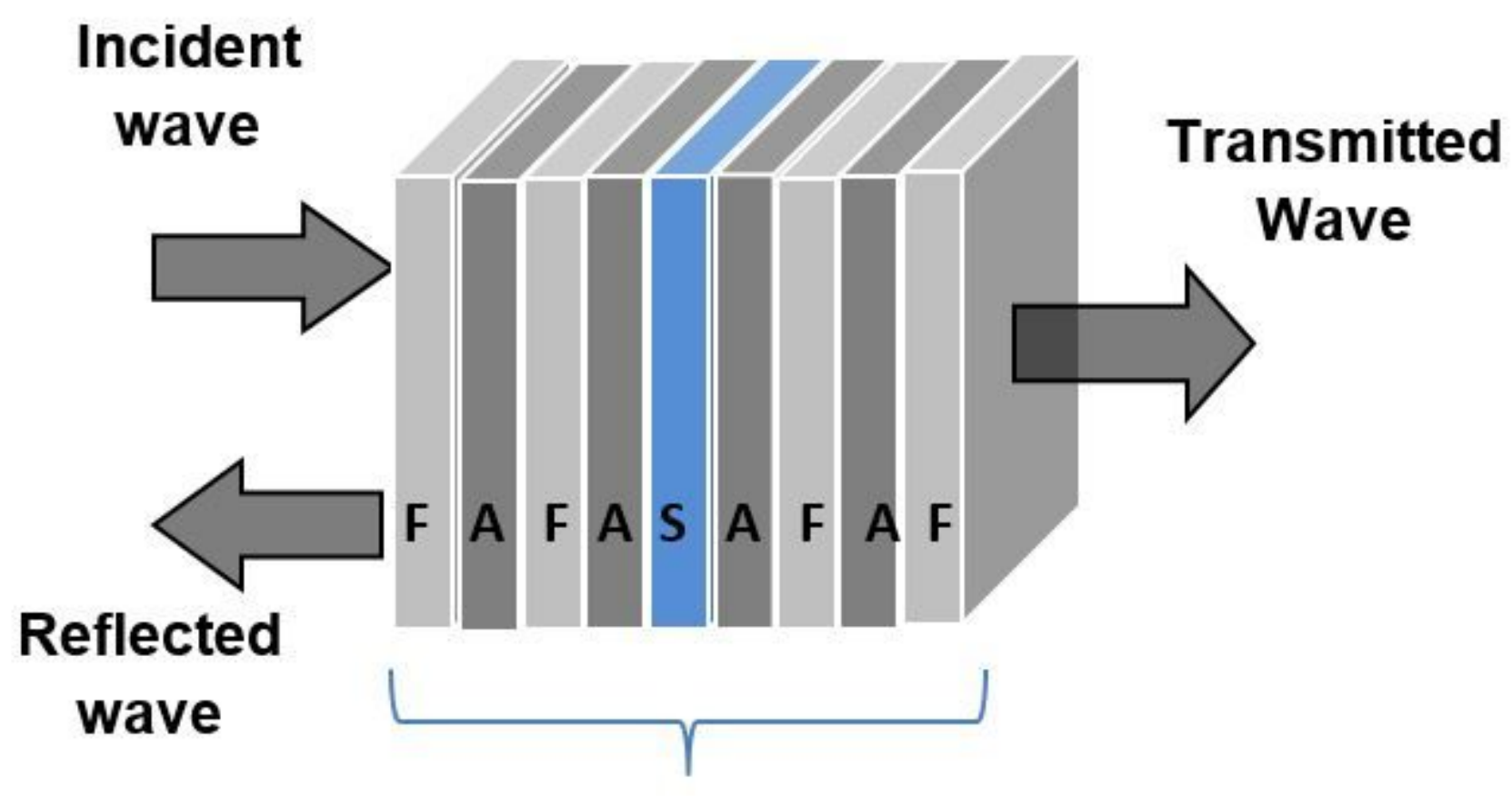

\section{P layers}

Figure 1

Schematic representation showing periodic photonic crystal with middle cavity containing the seawater, where $F$ is the fused silica layer, $A$ is the air layer and $S$ is the seawater layer. 


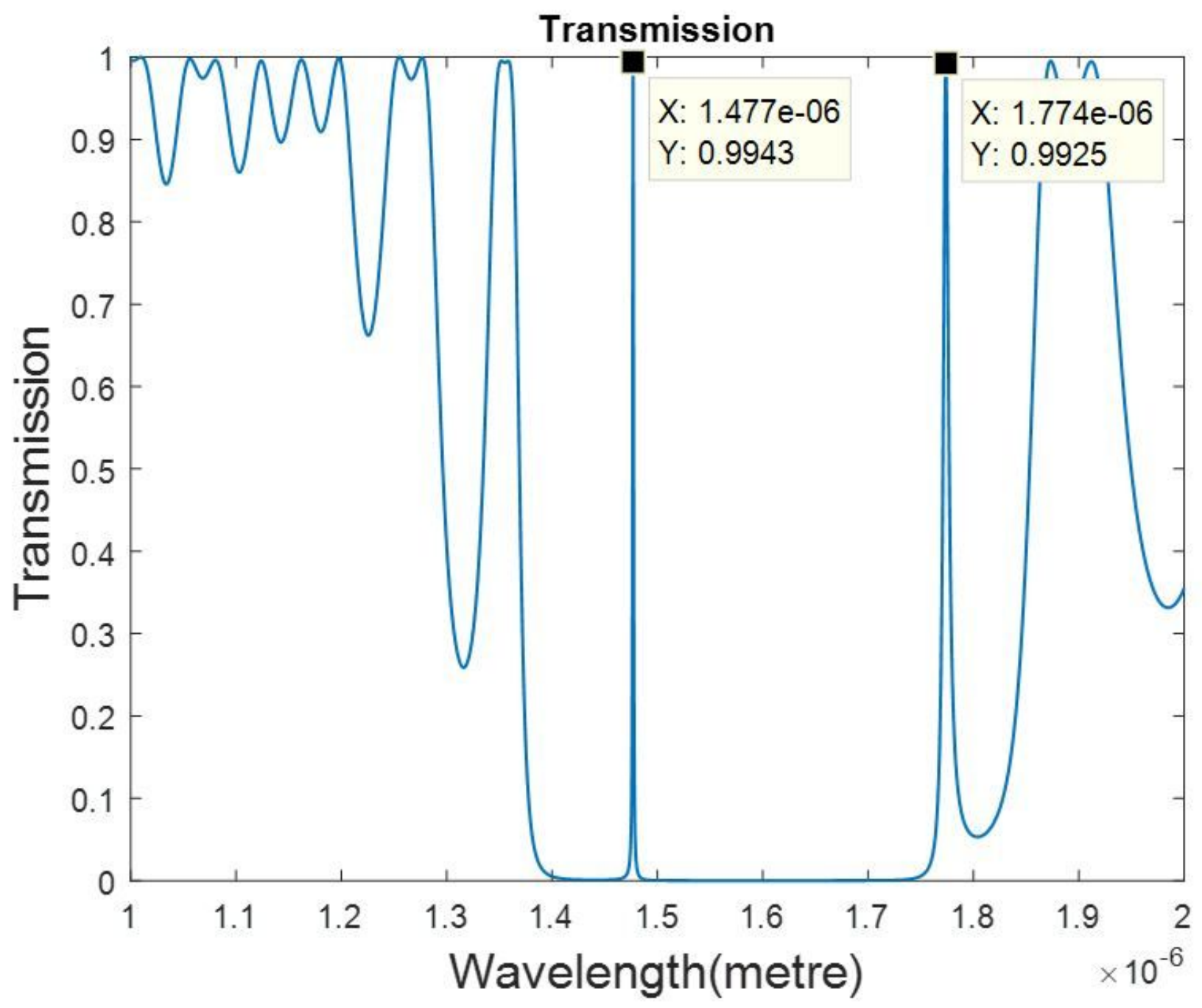

Figure 2

Transmittance spectrum for periodic photonic structure with 36 alternated layers of fused silica and air and with middle cavity filled by seawater have $50 \%$ salinity and at room temperature.
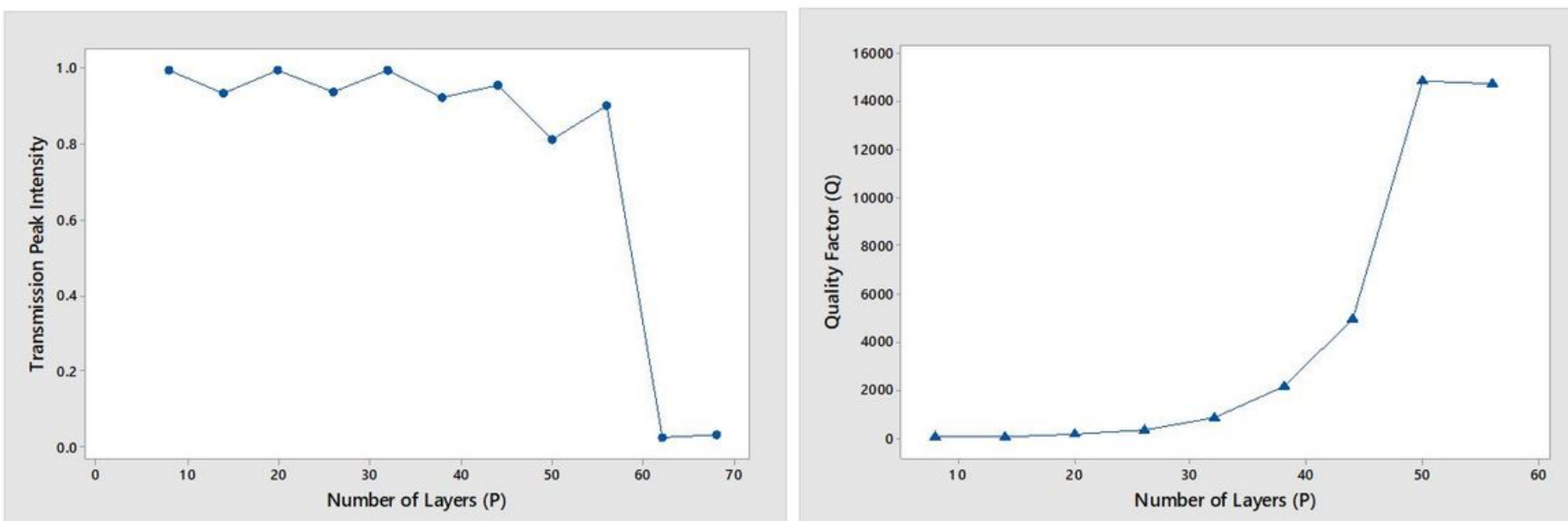

Figure 3 
Variation of the transmittance peak intensity and the quality factor as function of the layer number $(P)$ of photonic structure with P-alternated layers of fused silica and air and with middle cavity filled by seawater of $50 \%$ salinity and at room temperature.
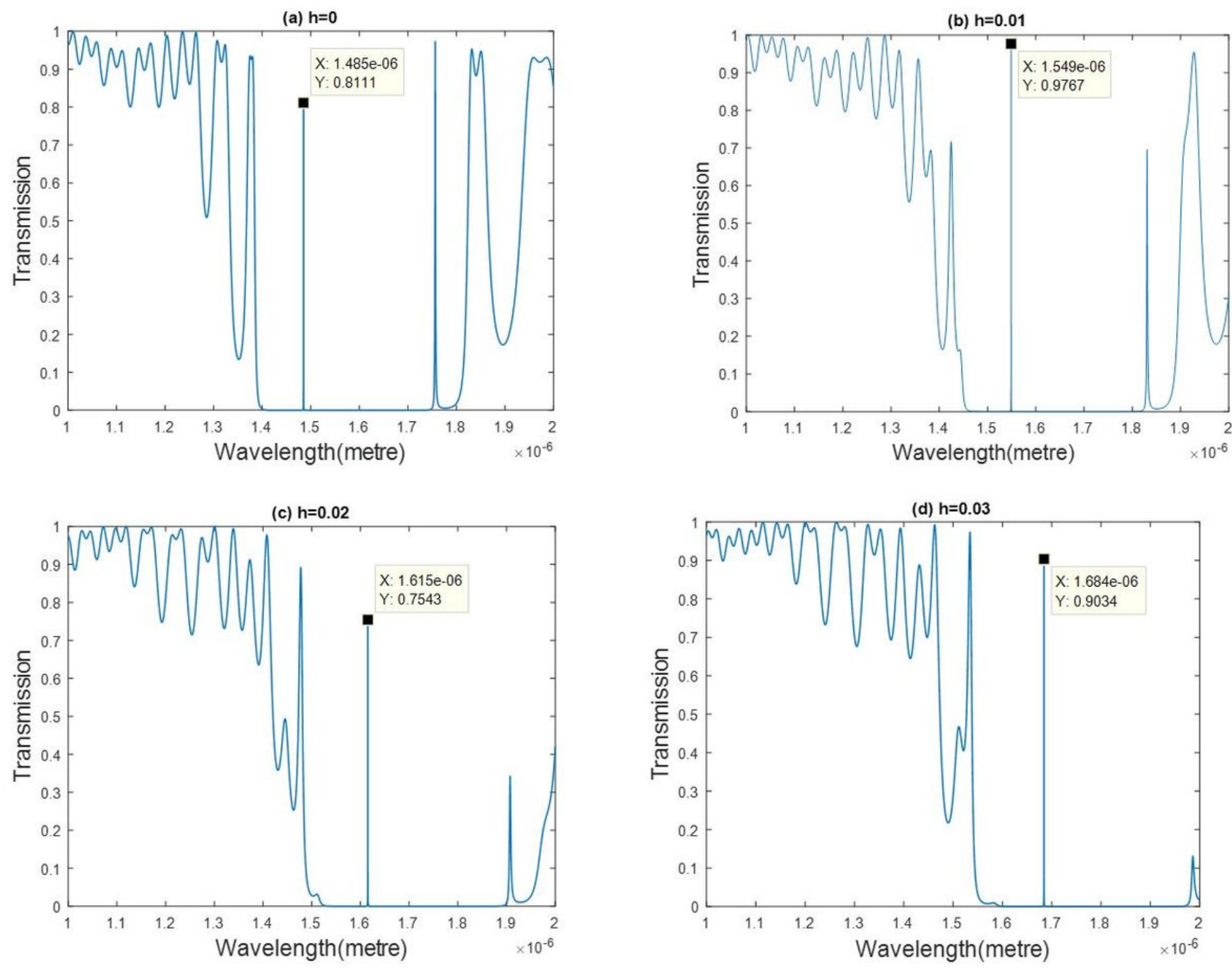

Figure 4

Variation of the transmittance peak intensity as function of the deformation degree $(\mathrm{h})$ for photonic structure with 50 -alternated layers of fused silica and air and with middle cavity filled by seawater of $50 \%$ salinity and at room temperature. 


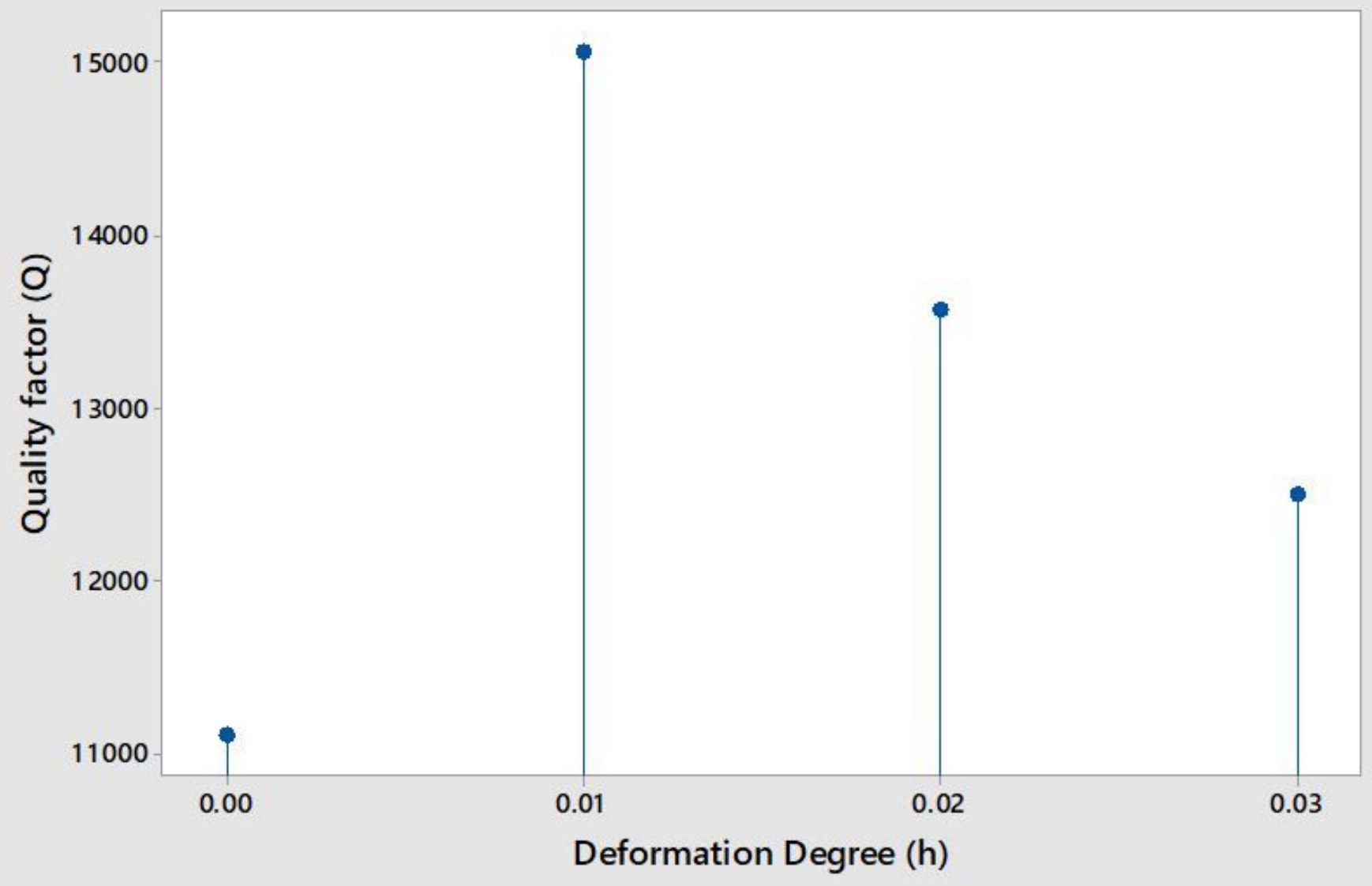

Figure 5

Variation of Q-factor as function of the deformation degree $(\mathrm{h})$ for photonic structure with 50 -alternated layers of fused silica and air and with middle cavity filled by seawater of $50 \%$ salinity and at room temperature. 


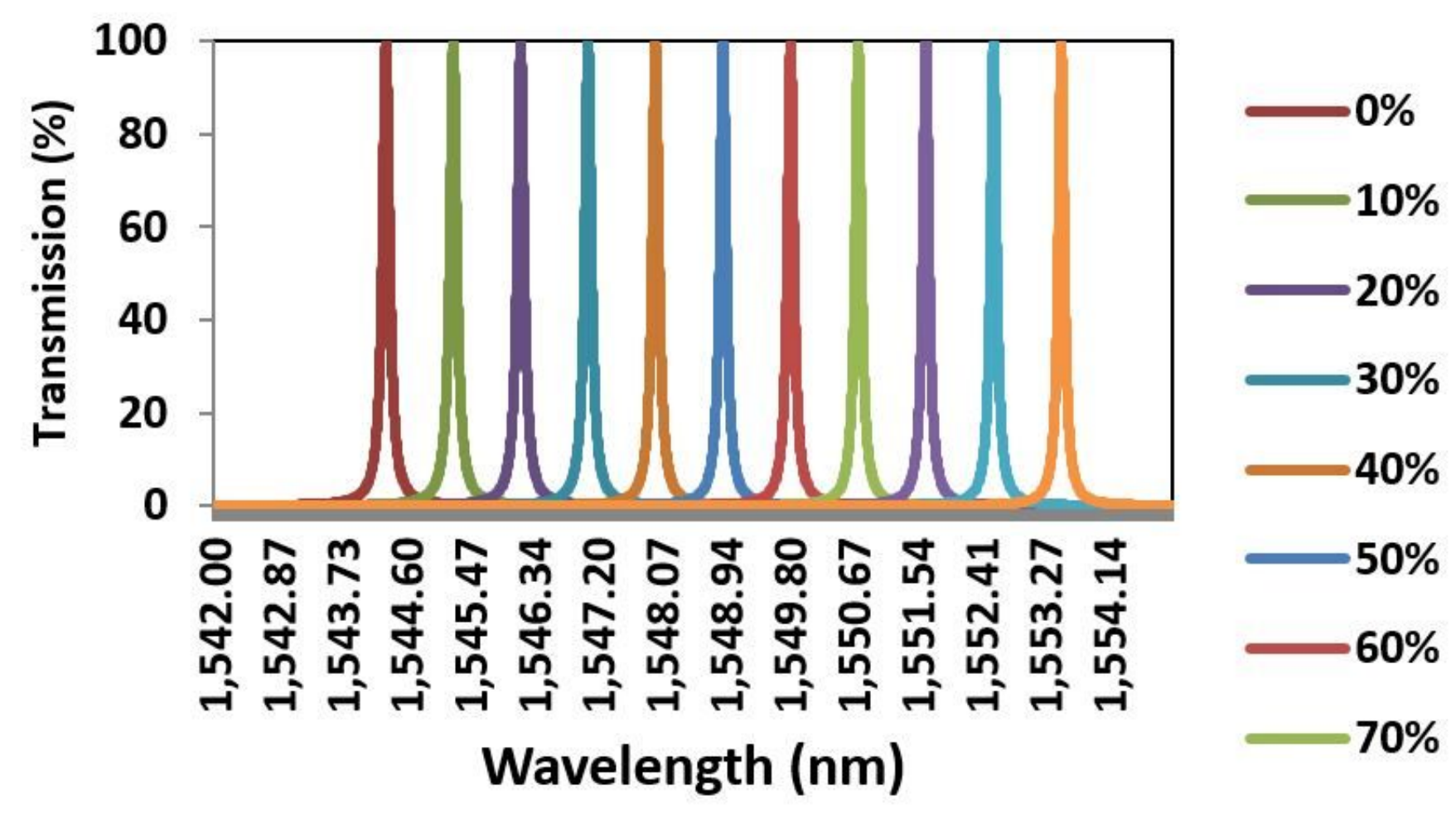

Figure 6

Optical transmittances as function of wavelength and salt level for deformed photonic structure with 50alternated layers of fused silica and air and with middle cavity filled by seawater at room temperature.

(a)

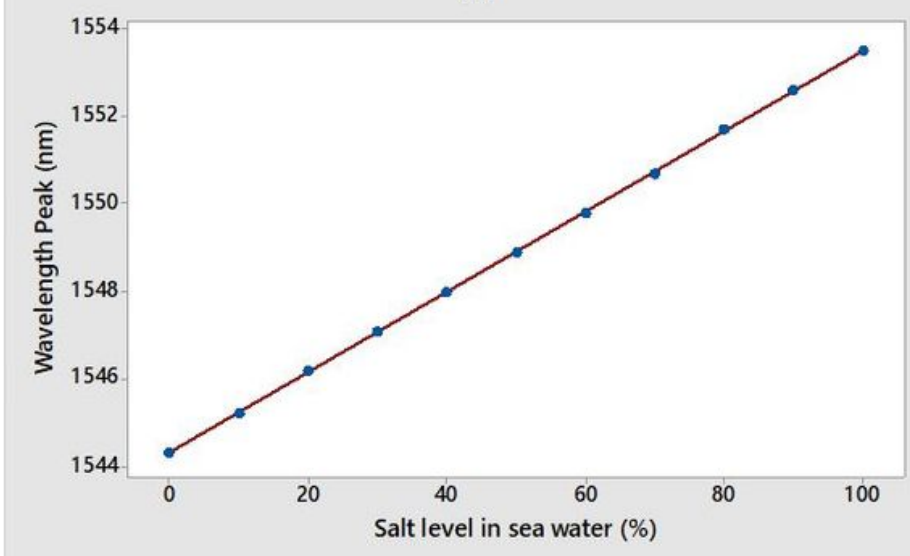

(b)

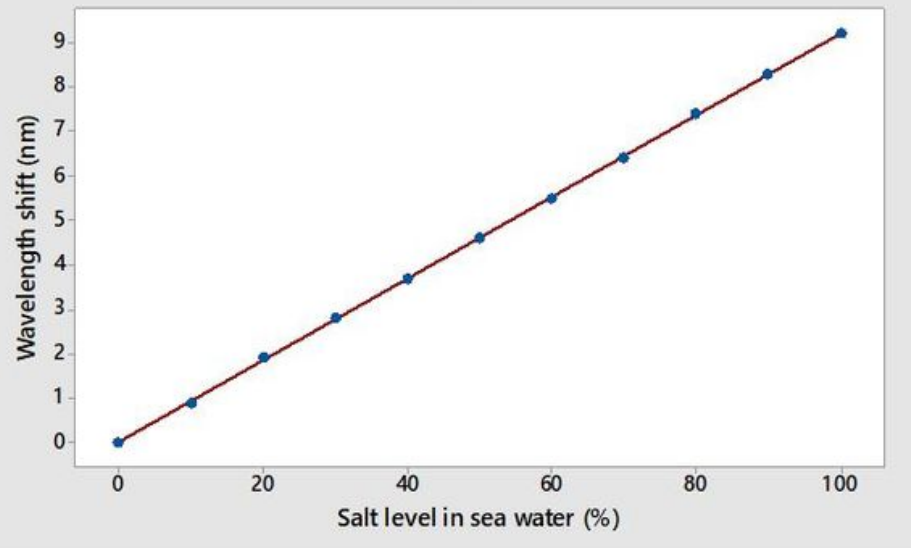

Figure 7

(a) Wavelength of Transmittance peak and (b) Peak wavelength shift as function of salt level for deformed photonic structure with 50 -alternated layers of fused silica and air and with middle cavity filled by seawater at room temperature. 


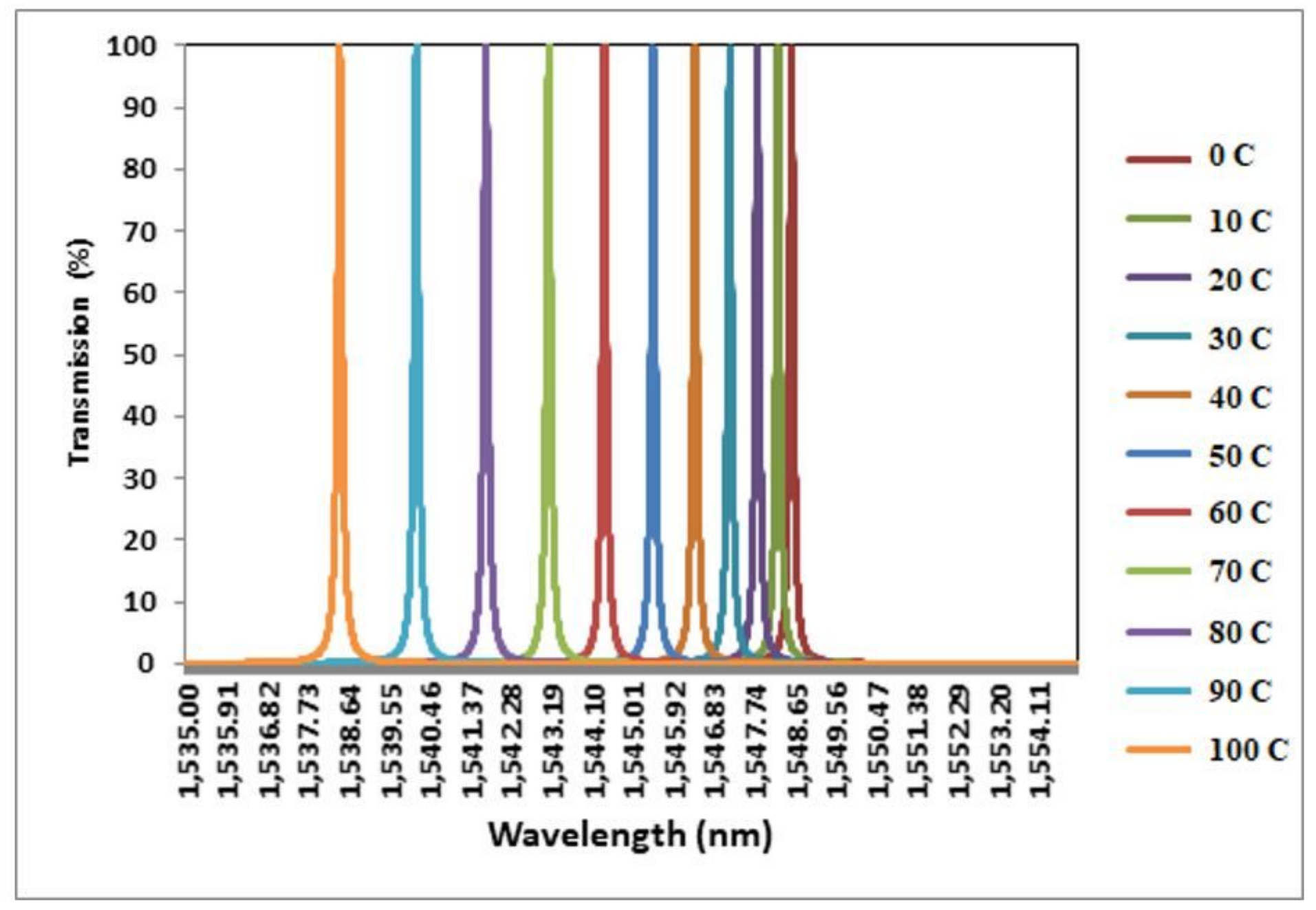

Figure 8

Optical transmittances as function of wavelength and water temperature degree ( $(\mathbb{)})$ for deformed photonic structure with 50-alternated layers of fused silica and air and with middle cavity filled by seawater at $35 \%$ salt level.
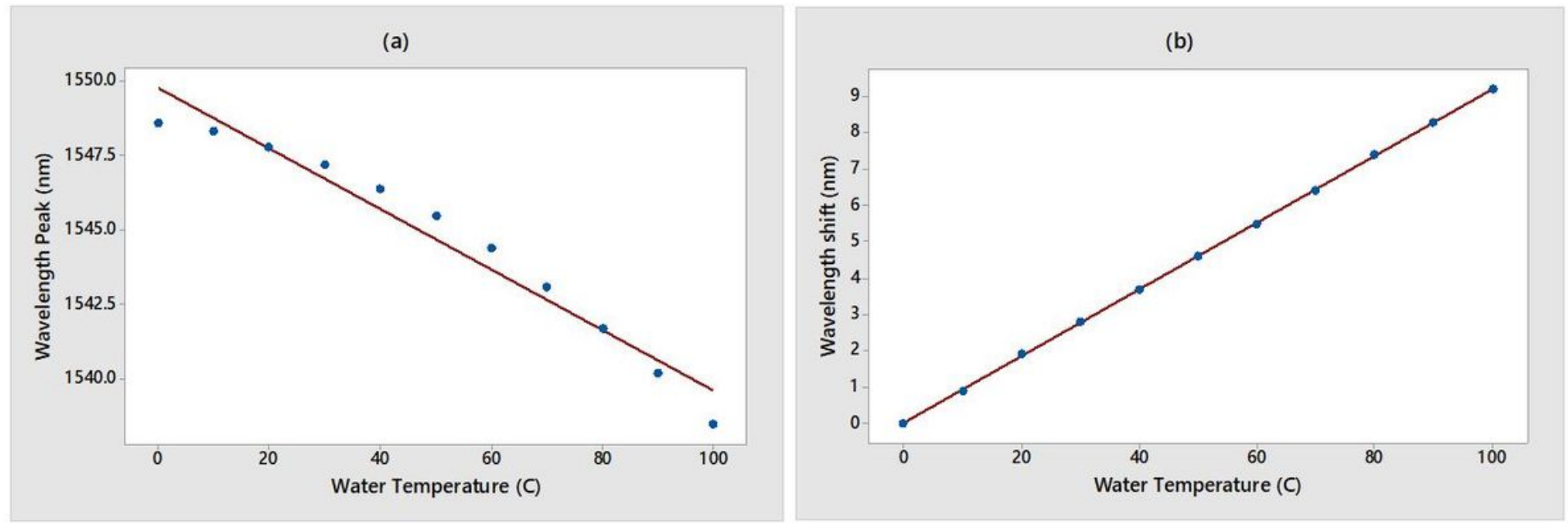

Figure 9 
(a) Wavelength of Transmittance peak and (b) Peak wavelength shift as function of water temperature degree $(\mathbb{\nabla})$ for deformed photonic structure with 50 -alternated layers of fused silica and air and with middle cavity filled by seawater at $35 \%$ salt level. 